\title{
Un nouveau groupe d'erreurs innées du métabolisme: les maladies peroxysomiales
}

Les peroxysomes sont des organites de distribution ubiquitaire, particulièrement abondants dans le foie et dans le rein. Les maladies altérant le nombre, la structure ou la fonction des peroxysomes sont nombreuses et graves ; les désordres biochimiques qu'elles entraînent et les symptomatologies associées renseignent sur la fonction réelle de ces organites. Ils semblent jouer, notamment, un rôle important dans la $\beta$-oxydation des acides gras à très longue chaîne et dans la synthèse des plasmalogènes, éthers phospholipidiques qui sont des constituants importants des membranes biologiques.

\section{Bwee Tien Poll-The Joseph Vamecq Jean-Pierre Draye Jean-Marie Saudubray}

\author{
ADRESSES \\ B.T. Poll-The: docteur en médecine. J.-M. \\ Saudubray: professeur au CHU Necker- \\ Enfants-Malades. Clinique de génétique \\ médicale et Inserm U.12, hôpital des Enfants- \\ Malades, 149, rue de Sèvres, 75743 Paris Cedex \\ 15, France. \\ J. Vamecq : docteur en médecine. J.-P. Draye : \\ docteur ès sciences. Laboratoire de chimie \\ physiologique, International Institute of Cel- \\ lular and Molecular Pathology (ICP), et uni- \\ versité catholique de Louvain (UCL), \\ Bruxelles, Belgique.
}

es peroxysomes sont des organites cellulaires sphériques ou ovoïdes de 0,1 à $1,5 \mu \mathrm{m}$ de diamètre, limités par une membrane trilaminaire (figure 1). Chez l'homme, on les observe essentiellement dans le foie et les reins, mais ils sont en fait présents dans la plupart des cellules sous forme d'organites plus petits ou microperoxysomes. Leur mise en évidence en microscopie optique nécessite une technique cytochimique particulière à la catalase. Leur morphologie détaillée n'est visible qu'en microscopie électronique. Durant de très nombreuses années, on a ignoré leur rôle biochimique chez l'homme, bien que Goldfischer ait signalé dès 1973 leur absence complète dans le foie de malades présentant un syndrome polymalformatif très caractéristique, le syndrome de Zellweger. La découverte qu'une anomalie de fonctionnement du peroxysome pouvait être associée à des maladies génétiques a ouvert une voie de recherche nouvelle. Un nouveau groupe d'erreurs innées du métabo- lisme a été découvert qui a contribué grandement à la compréhension du rôle physiologique des peroxysomes.

\section{La biochimie du peroxysome}

Les protéines peroxysomiales sont synthétisées par les ribosomes libres. La plupart des proenzymes peroxysomiales (protéines natives présentes dans le cytosol) sont très semblables sinon identiques aux enzymes peroxysomiales matures. Les peroxysomes se multiplient par simple division et ne possèdent pas d'ADN. Récemment, les trois protéines de la $\beta$-oxydation peroxysomiale (acylCoA oxydase, enzyme bifonctionnelle et 3-cétothiolase) ont été clonées à partir du foie de rat, ce qui devrait permettre de très rapides progrès dans la compréhension de la pathologie moléculaire des peroxysomes. Dès 1960, De Duve montrait que la respiration peroxysomiale consiste en l'oxydation de nombreux substrats (lactate, glycolate, urate, acides aminés $\mathrm{D}$, acylCoAs, etc.), par désoxyda- 
tion, avec production d'eau oxygénée décomposée en eau et oxygène par la catalase ([1]; figure 3). A l'heure actuelle, on connaît de très nombreuses fonctions biochimiques du peroxysome chez l'homme, fonctions dont la liste ne cesse de s'allonger et dont les principales sont rassemblées dans le Tableau I et la figure 2.

La $\beta$-oxydation peroxysomiale des acides gras diffère schématiquement de la $\beta$-oxydation mitochondriale par les points suivants: (l) entrée des acides gras dans le peroxysome indépendante de la carnitine après une activation par une acyl-CoA ligase; (2) spécialisation dans la dégradation des acides gras à chaîne très longue $\left(>\mathrm{C}_{22}\right)$, notamment de l'acide lignocérique en $\mathrm{C}_{24}$ et de l'acide cérotique en $\mathrm{C}_{26}$ [2] ; (3) possibilité de raccourcir les acides dicarboxyliques à chaîne longue et moyenne produits par l' $\omega$ oxydation microsomale[3]; (4) incapacité à raccourcir les chaînes carbonées d'acides gras

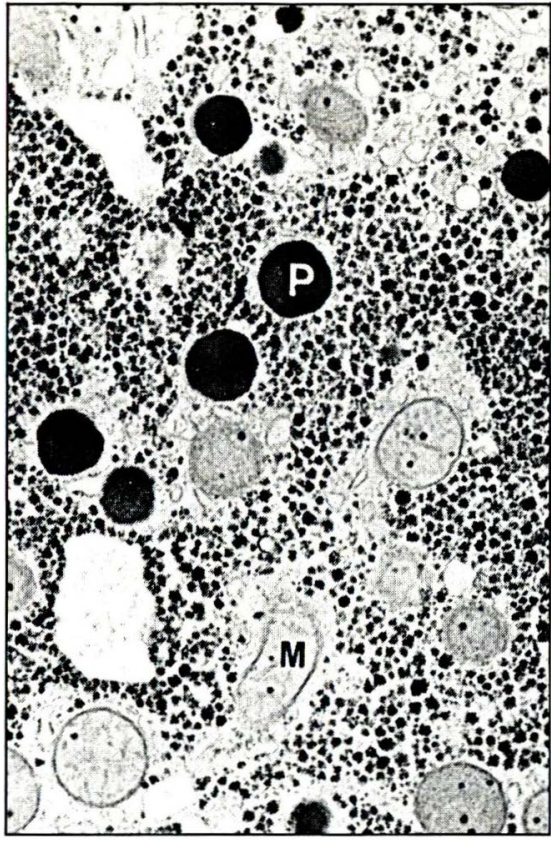

Figure 1. Hépatocyte: présence de peroxysomes $(P)$ mis en évidence par la réaction de la catalase peroxysomiale. $M=$ mitochondrie. (Pr Roels, Bruxelles).

mono- ou dicarboxyliques au-dessous de $\mathrm{C}_{6} ;(5)$ possibilité de dégrader les acides gras mono- et polyinsaturés grâce à une isomérase capable de déplacer la double liaison [4]; (6) première étape de déshydrogénation par une acyl-CoA oxydase couplée à la catalase (au lieu d'une acylCoA déshydrogénase à FAD); (7) deuxième et troisième étapes de la $\beta$-oxydation (hydratation et déshydrogénation à NAD) effectuées par une seule et même enzyme dite enzyme bifonctionnelle; (8) quatrième étape catalysée par une 3 . cétothiolase peroxysomiale.

De nombreuses voies biochimiques, telles celles du catabolisme de l'acide pipécolique ou de l'acide phytanique, sont probablement peroxysomiales mais non identifiées. Le rôle physiologique de la $\beta$-oxydation peroxysomiale et ses relations avec la $\beta$-oxydation mitochondriale sont encore presque inconnus chez l'homme. La plupart des substances inductrices des peroxysomes chez le rat n'exerce pas cet effet chez

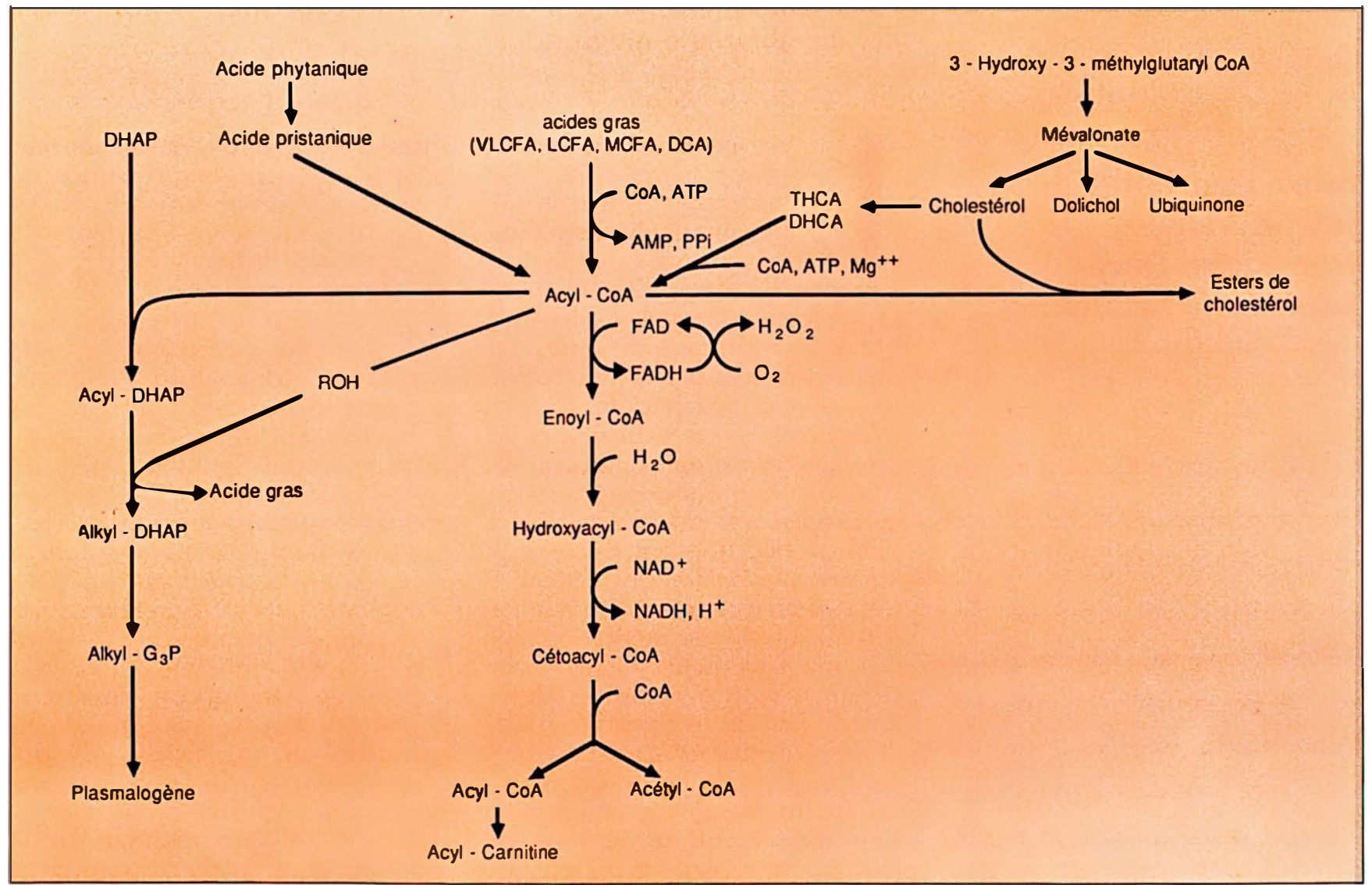

Figure 2. Voies métaboliques simplifiées dans le peroxysome. VLCFA = acide gras à très longue chaîne; LCFA = acide gras à longue chaîne; MCFA = acide gras à moyenne chaîne; DCA = acide dicarboxylique ; THCA VDCA = acides di-et trihydroxycoprostanoïques; $D H A P=$ dihydroxyacétone phosphate; G3P = glycérol-3-phosphate; $R O H=$ alcool gras. 


\begin{tabular}{|c|c|c|}
\hline \multicolumn{3}{|c|}{$\begin{array}{l}\text { Tableau I } \\
\text { PRINCIPALES FONCTIONS BIOCHIMIQUES DES PEROXYSOMES } \\
\text { DONT LA DÉFICIENCE A ÉTÉ OBSERVÉE CHEZ L'HOMME }\end{array}$} \\
\hline Fonction & Enzyme & $\begin{array}{l}\text { Conséquence métabolioque } \\
\text { en cas de déficit }\end{array}$ \\
\hline $\begin{array}{l}\text { Métabolisme du } \\
\text { peroxyde d'hydrogène } \\
\left(\mathrm{H}_{2} \mathrm{O}_{2}\right)\end{array}$ & Catalase & Acatalasémie \\
\hline $\begin{array}{l}\text { B-oxydation } \\
\text { des acides gras } \\
\text { à chaîne très longue } \\
\text { (AGTLC) }\end{array}$ & $\begin{array}{l}\text { Lignoceryl-CoA ligase } \\
\text { Acyl-CoA oxydase } \\
\text { Enzyme bifonctionnelle } \\
\text { 3-cétothiolase }\end{array}$ & $\begin{array}{l}\text { Accumulation d'acides gras à } \\
\text { très longue chaîne (AGTLC) }\end{array}$ \\
\hline $\begin{array}{l}\beta \text {-oxydation des } \\
\text { acides dicarboxyliques }\end{array}$ & Idem & $\begin{array}{l}\text { Accumulation d'acides dicar- } \\
\text { boxyliques à chaîne longue et } \\
\text { moyenne }\end{array}$ \\
\hline $\begin{array}{l}\text { Métabolisme } \\
\text { des acides biliaires }\end{array}$ & $\begin{array}{l}\text { Acyl-CoA ligase spécifi- } \\
\text { que : } \beta \text {-oxydation }\end{array}$ & $\begin{array}{l}\text { Accumulation d'acides dihy- } \\
\text { droxy et trihydroxy coprosta- } \\
\text { niques (DHC, THC): précur- } \\
\text { seur de l'acide cholique et } \\
\text { chénodéoxycholique }\end{array}$ \\
\hline $\begin{array}{l}\text { Synthèse } \\
\text { des plasmalogènes }\end{array}$ & $\begin{array}{l}\text { Acyl-CoA: dihydroxya- } \\
\text { cétone phosphate acyl- } \\
\text { transférase (DHAP-AT) } \\
\text { Alkyldihydroxyacétone } \\
\text { phosphate synthase } \\
\text { (alkyl DHAP synthase) }\end{array}$ & $\begin{array}{l}\text { Défaut de synthèse des plas- } \\
\text { malogènes }\end{array}$ \\
\hline $\begin{array}{l}\text { Oxydation de l'acide } \\
\text { phytanique? }\end{array}$ & $\begin{array}{l}\text { Acide phytanique oxy- } \\
\text { dase }\end{array}$ & $\begin{array}{l}\text { Accumulation d'acide phyta- } \\
\text { nique }\end{array}$ \\
\hline $\begin{array}{l}\text { Oxydation de l'acide } \\
\text { pipécolique }\end{array}$ & $\begin{array}{l}\text { Acide pipécolique oxy- } \\
\text { dase }\end{array}$ & $\begin{array}{l}\text { Accumulation d'acide pipéco- } \\
\text { lique }\end{array}$ \\
\hline $\begin{array}{l}\text { Catabolisme du } \\
\text { glyoxylate }\end{array}$ & $\begin{array}{l}\text { Alanine glyoxylate } \\
\text { amino transférase }\end{array}$ & Dépôts d'oxalate de calcium \\
\hline $\begin{array}{l}\text { Synthèse du cholesté- } \\
\text { rol }\end{array}$ & $\begin{array}{l}\text { 3-hydroxy-3-methyl- } \\
\text { glutaryl-CoA } \\
\text { (HMG-CoA) réductase }\end{array}$ & $?$ \\
\hline
\end{tabular}

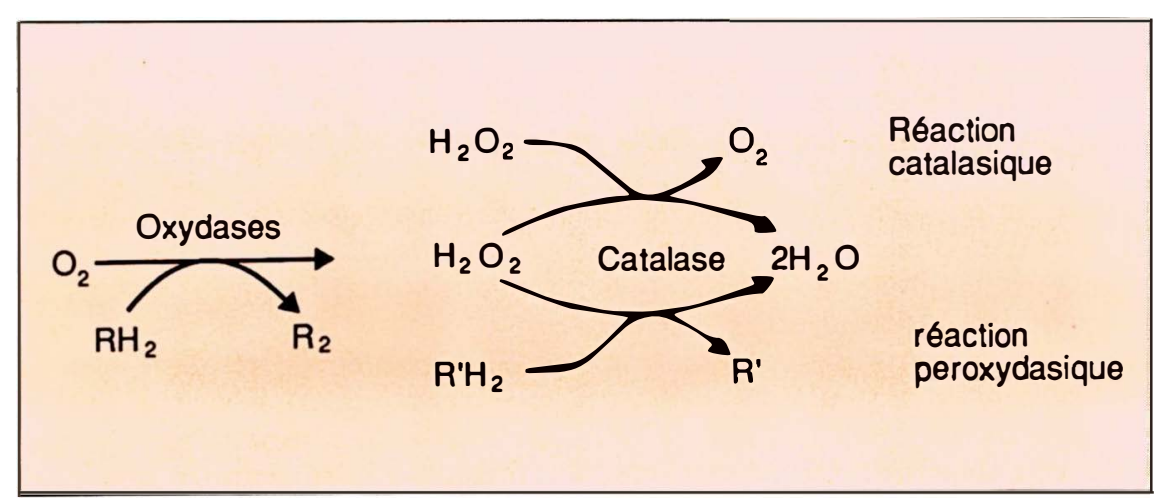

Figure 3. Peroxysomes et métabolisme de l'eau oxygénée. Les oxydases oxydent le substrat $R$ en formant de l'eau oxygénée. $\mathrm{H}_{2} \mathrm{O}_{2}$ peut être transformée par la catalase, libérant de l'oxygène moléculaire et oxydant un substrat $R^{\prime} H_{2}$.

$\mathrm{m} / \mathrm{s} n^{\circ} 9$ vol. 4, novembre 88 l'homme (notamment les hypocholesterolémiants type «clofibrate»). Cependant, on sait que le jeûne, le catabolisme, et les régimes riches en phytol et acides gras à chaîne longue multiplient le nombre des peroxysomes hépatiques. Il est probable que le peroxysome joue un rôle dans la synthèse endogène du cholestérol, puisqu'on a démontré chez le rat traité par la cholestyramine que 20 à $30 \%$ de l'activité totale de HMG$\mathrm{CoA}^{*}$ réductase hépatique étaient localisés dans le peroxysome [5].

Les étapes clés de la synthèse des plasmalogènes, centrées sur le dihydroxyacétone phosphate (DHAP) c'est-à-dire l'acyl-CoA : dihydroxyacétone phosphate acyltransférase (DHAP-AT) et l'alkyl DHAP synthase sont principalement localisées dans les peroxysomes [6]. Les plasmalogènes représentent les produits terminaux de synthèse des éthers phospholipidiques et sont des constituants importants des membranes. A l'exception du facteur activateur de l'agrégation plaquettaire, le rôle biologique des éthers lipidiques reste encore largement incompris.

\section{Les désordres peroxysomiaux primaires}

Les maladies héréditaires du métabolisme des peroxysomes peuvent être classées en quatre catégories définies par le nombre de déficits enzymatiques observés (Tableau II). Cette classification est évidemment provisoire car, pour la plupart des entités jusqu'ici décrites, le déficit moléculaire précis n'a pas encore été identifié.

Groupe I : affections à présentation neurologique et déficits biochimiques multiples. Les perturbations métaboliques dans les maladies classées en groupe I résultent apparemment d'un défaut de fabrication de l'enveloppe peroxysomiale ou d'un défaut de l'entrée de protéines dans le peroxysome. La plupart des fonc-

* Hydroxyméthylglutaryl-CoA réductase. 


\section{RÉFÉRENCES}

1. De Duve C, Beaufay H, Jacques P, et al. Intracellular distribution of catalase and of some oxidases in rat liver. Biochim Biophys Acta $1960 ; 40$ : 186-7.

2. Singh H, Derwas N, Poulos A. $\beta$-Oxidation of very-long-chain fatty acids and their coenzyme A derivates by human skin fibroblasts. Arch Biochem Biophys 1987 ; 354 : 526 33.

3. Vameca J, Draye JP. Interactions between the $\omega$-and the $\beta$-oxidations of fatty acids. $J$ Biochem (Tokyo) 1987 ; 102 : 225-34.

4. Kärki T, Hakkola E, Hassinen IE, Hitunen JK. $\beta$-Oxidation of polyunsaturated fatty acids in peroxisomes. Subcellular distribution of delta-3, delta-2-enoyl-CoA isomerase activity in rat liver. FEBS Lett 1987 ; 215 : 228 32.

5. Keller GA, Pazirandeh M, Krisans SK. 3-Hydroxy-3-methylglytaryl-coenzyme A reductase localization in rat liver peroxisomes and microsomes of control and cholestyramine-treated animals: quantitative biochemical and immunoelectron microscopical analysis. J Cell Biol 1986; 103 : 875-86.

6. Hajra AK, Bishop ME. Glycerolipid biosynthesis in peroxisomes via the acyl dihydroxyacetone phosphate pathway. Ann NY Acad Sci 1982 ; 386 : 170-81.

7. Goldfischer S, Moore CL, Johnson AB, et al. Peroxisomal and mitochondrial defects in the cerebro-hepato-renal syndrome. Science $1972 ; 182: 62-4$

8. Schutgens RBH, Heymans HSA, Wander RJA, van den Bosch H, Tager JM. Peroxisomal disorders : a newly recognised group of genetic diseases. Eur J Pediatr 1986 ; 144 : 430 40

9. Santos MJ, Imanaka T, Shio $H$, Small GM, Lazarow PB. Peroxisomal membrane ghosts in Zellweger syndrome. Aberrant organelle assembly. Science $1988 ; 239$ : 1536-8.

10. Kelley RI, Datta NS, Dobyns WB, et al. Neonatal adrenoleukodystrophy: new cases, biochemical studies and differentiation from Zellweger and related peroxisomal polydystrophy syndromes. Am J Med Genet 1986 23 : 869-901.

11. Goldfischer S, Collins H, Rapin I, et al. Peroxisomal defects in neonatal onset and X linked adrenoleukodystrophy. Science 1985 ; 227 : 67-70.

12. Tager JM. Inborn errors of cellular organelles : an overview. J Inher Metab Dis 1987 10 (suppl. 1) : 3-10.

13. Poll-The BT, Skjeldal $\mathrm{OH}$, Stokke $\mathrm{O}$ Poulos A, Demaugre F, Saudubray JM. Phytanic acid $\alpha$-oxidation and complementation analysis of classical Refsum and peroxisomal disorders. Hum Genet (sous presse)

14. Poll-The BT, Saudubray JM, Ogier HAM, et al. Infantile Refsum disease: an inherited peroxisomal disorder. Comparison with Zellweger syndrome and neonatal adrenoleukodystrophy. Eur J Pediatr 1987; 146 : 477-83.

15. Roels F, Cornelis A, Poll-The BT, et al. Hepatic peroxisomes are deficient in infantile Refsum's disease: a cytochemical study of 4 cases. Am J Med Genet $1986 ; 25: 257-71$.

\begin{tabular}{|c|c|c|c|}
\hline & \multicolumn{3}{|c|}{$\begin{array}{c}\text { Tableau \| } \\
\text { CLASSIFICATION DES MALADIES HÉRÉDITAIRES PEROXYSOMIALES }\end{array}$} \\
\hline & Maladie & Déficit enzymatique & $\begin{array}{l}\text { Peroxysomes } \\
\text { hépatiques }\end{array}$ \\
\hline $\mathbf{I}$ & $\begin{array}{l}\text { Zellweger } \\
\text { ALDN } \\
\text { Refsum infantile } \\
\text { Zellweger-like }\end{array}$ & $\begin{array}{l}\text { Multiple } \\
\text { Multiple ; } \\
\text { Protéine } \\
\text { bifonctionnelle? } \\
\text { Multiple } \\
\text { Multiple }\end{array}$ & $\begin{array}{l}\text { Absents ou très réduits } \\
\text { Normaux }\end{array}$ \\
\hline II & $\begin{array}{l}\text { Chondrodysplasie } \\
\text { rhizomélique }\end{array}$ & $\begin{array}{l}\text { DHAP-aryltransférase } \\
\text { Alkyl-DHAP synthase } \\
\text { Phytanique oxydase }\end{array}$ & Anormaux \\
\hline III & $\begin{array}{l}\text { ALD liée à I'X } \\
\text { Pseudo-ALDN } \\
\text { Pseudo-Zellweger } \\
\text { Acidémie pipécolique } \\
\text { «ALD-variante» } \\
\text { (Refsum classique) }\end{array}$ & $\begin{array}{l}\text { AGTLC-CoA ligase } \\
\text { Acyl-CoA oxydase } \\
\text { 3-cétothiolase } \\
\text { Pipécolique oxydase } \\
\text { AGTLC-CoA ligase? } \\
\text { Phytanique oxydase }\end{array}$ & $\begin{array}{l}\text { Normaux } \\
\text { Anormaux } \\
\text { Anormaux } \\
\text { Anormaux } \\
\text { Anormaux } \\
\text { Inconnus }\end{array}$ \\
\hline IV & $\begin{array}{l}\text { Hyperoxalurie type I } \\
\text { Acatalasémie }\end{array}$ & $\begin{array}{l}\text { Alanine : glyoxylate } \\
\text { aminotransférase } \\
\text { Catalase }\end{array}$ & $\begin{array}{l}\text { Normaux } \\
\text { Inconnus }\end{array}$ \\
\hline
\end{tabular}

$A L D=$ adrénoleucodystrophie $: A L D N=$ adrénoleucodystrophie néonatale $;$ DHAP $=$ dihydroxyacétone phosphate $; A T=$ acyltransférase ; $A G T L C=$ acide gras à très longue chaîne.

tions biochimiques décrites dans le Tableau I sont déficientes.

- Le syndrome cérébro-hépato-rénal de Zellweger (SZ). Ce syndrome polymalformatif autosomique récessif représente le prototype des désordres peroxysomiaux à déficiences enzymatiques multiples. Les patients atteints ont dès la naissance une dysmorphie faciale typique (figure 4), une hypotonie musculaire massive, des calcifications épiphysaires (figure $5)$, des convulsions dès les premiers jours de vie et ne font aucune acquisition psychomotrice. Une hépatomégalie, une surdité, des troubles visuels (rétinite pigmentaire, atrophie optique, cataracte) et une atteinte surrénalienne apparaissent durant les premiers mois de la vie. Il existe fréquemment des kystes rénaux. L'atteinte du système nerveux central est caractérisée par un trouble de la migration neuronale du cortex et de la région sous-corticale et par une démyélinisation de la substance blanche. La plupart des patients décèdent avant l'âge de six mois.

Les peroxysomes hépatiques et rénaux sont morphologiquement absents [7].

Biochimiquement, la plupart des enzymes peroxysomiales sont sévèrement déficientes, ce qui entraîne notamment une accumulation sérique d'acides gras à très longue chaîne (AGTLC), acides biliaires, pipécolique et phytanique, ainsi qu'un déficit des plasmalogènes [8]. Le défaut primaire dans le SZ demeure inconnu. Il semble que la membrane peroxysomiale soit normalement synthétisée bien que non visualisable, et qu'il existe un défaut du mécanisme d'entrée des protéines dans le peroxysome [9]. Seules quelques enzymes peroxysomiales peuvent demeurer fonctionnelles dans le cytosol, notamment la catalase, la Daminoacide oxydase, la L- $\alpha$-hydroxyacide oxydase et l'alanine glyoxylate aminotransférase

- L'adrénoleucodystrophie néonatale $(A L D N)$. L'ALDN est une maladie récessive autosomique, génétiquement, cliniquement et anatomopathologiquement différente de l'ALD juvénile. L'aspect clinique et les anomalies biochimiques ressem- 
blent à ce qui est observé dans le SZ. Cependant l'ALDN apparaît cliniquement comme une affection hétérogène. La dysmorphie faciale n'est pas caractéristique et n'est pas toujours présente [10]. Certains malades présentent une hypotonie dès la naissance, des convulsions dès les premiers jours de la vie, une rétinite pigmentaire et/ou une atrophie optique, une surdité, une hépatomégalie, une atteinte surrénalienne et décèdent avant un an. D'autres malades sont moins sévèrement atteints dans les premiers mois de vie et peuvent présenter un certain développement avant leur détérioration progressive et la mort qui survient habituellement avant l'âge de six ans.

Le cerveau présente une démyélinisation souvent plus importante que celle observée dans le $\mathrm{SZ}$; il existe parfois quelques défauts de migration neuronale, mais moins importants que dans le SZ. Il n'y a ni calcification épiphysaire, ni kyste rénal comme dans le SZ.

La différence la plus marquée est que les peroxysomes ne sont habituellement pas absents du foie ni du rein, même si parfois ils sont beaucoup moins nombreux et plus petits qu'à l'ordinaire [11].

Bien que les anomalies biochimiques soient semblables à celles observées dans le $S Z$, il s'agit certainement d'une affection différente, comme en témoignent notamment les études de complémentation cellulaire [12, 13].

- La maladie de Refsum infantile (RI). La maladie de RI est totalement différente de la maladie de Refsum classique qui est due à un déficit isolé en acide phytanique $\alpha$-hydroxylase, débute plus tardivement, évolue lentement et ne comporte jamais de retard mental. Le RI ne comporte à la période néonatale que peu ou pas de signes cliniques. Les premiers signes apparaissent dans la première année de la vie et associent gros foie, troubles digestifs, hypocholestérolémie, cassure de croissance, simulant une malabsorption intestinale. Puis apparaissent un retard psychomoteur avec hypotonie modérée, une rétinite pigmentaire, une surdité, une dysmorphie faciale mineure, des troubles du comportement et des signes biologiques d'insuffisance surrénalienne. La plupart de ces $\mathrm{m} / \mathrm{s} n^{\circ} 9 \mathrm{vol}$. 4, novembre 88

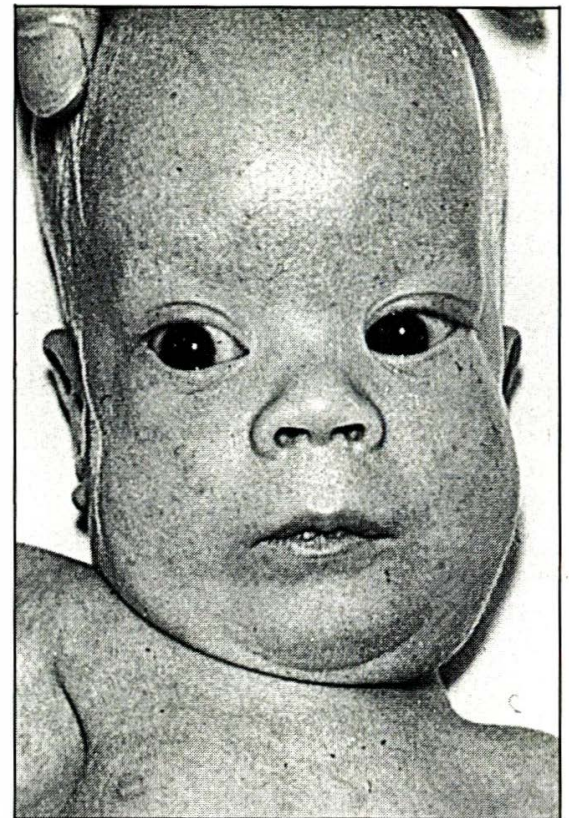

Figure 4. Syndrome de Zellweger: dysmorphie faciale.

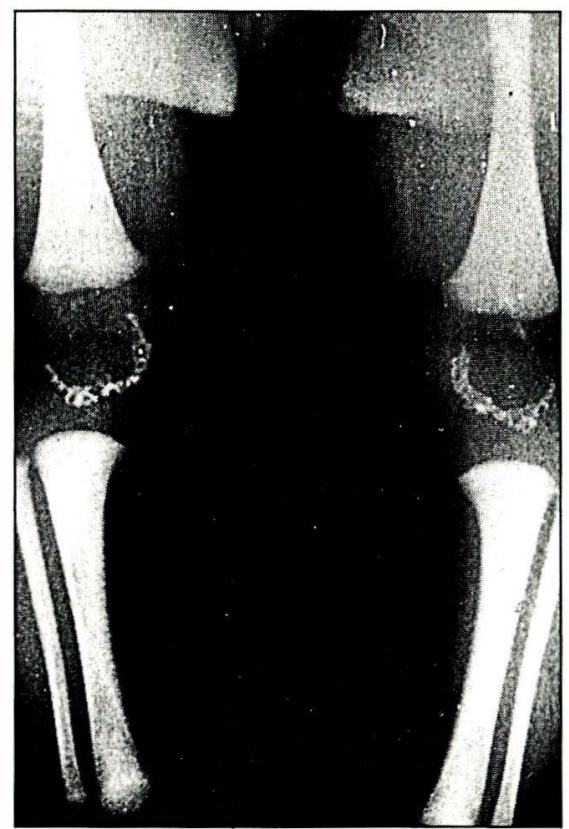

Figure 5. Syndrome de Zellweger: calcifications épiphysaires.

enfants sont vivants au-delà de dix ans [14].

Le cerveau ne montre en général pas de malformation.

Chez la plupart des malades, les peroxysomes sont morphologique- ment absents au niveau du foie [15]. Bien que les présentations clinique et neuroanatomopathologique soient différentes de celles du $\mathrm{SZ}$, biochimiquement rien ne distingue le SZ du RI. Le SZ et le RI font partie du même groupe de complémentation génétique, tandis que l'ADLN fait partie d'un autre groupe [12, 13]. Les différences d'expression clinique demeurent actuellement inexpliquées.

- "Zellweger-like " syndrome. Deux malades ont été décrits avec un phénotype clinique comparable à celui de SZ, mais les peroxysomes hépatiques sont présents en nombre normal [16]. Dans ce désordre, il existe apparemment un défaut de l'entrée de protéines de la $\beta$-oxydation peroxysomiale dans le peroxysome.

- Le diagnostic anténatal. Dans le SZ, l'ADLN et la maladie de RI, le diagnostic anténatal est possible sur prélèvement de trophoblastes et/ou amniocytes par l'étude des acides gras à très longue chaîne, l'activité de l'acide phytanique $\alpha$-oxydase, la synthèse des plasmalogènes et la mesure de la catalase particulaire et sa libération lors des fractionnements cellulaires à la digitonine [17, 18]. Ces méthodes ne permettent pas de distinguer ces affections l'une de l'autre.

Groupe II : ce groupe est représenté actuellement par une seule entité comportant trois déficits biochimiques.

- La chondrodysplasie ponctuée de type rhizomélique. Cette maladie récessive autosomique, connue de longue date, est caractérisée par un nanisme prédominant aux parties proximales des membres, une dysmorphie faciale, une cataracte, des contractures articulaires, des calcifications épiphysaires et un retard psychomoteur sévère.

Biochimiquement, il existe un déficit en DHAP-AT et Alkyl DHAP synthase rendant compte du défaut de synthèse des plasmalogènes associé à un déficit de l'activité de l'acide phytanique $\alpha$-oxydase [19]. Dans ce désordre, la $\beta$-oxydation peroxysomiale est normale, ce qui explique l'absence d'accumulation des acides gras à très longue chaîne et des acides diet tri-hydroxycoprostanoïques. 


\section{RÉFÉRENCES}

16. Suzuki Y, Shimozawa N, Orii T, Igarashi $\mathrm{N}$, Kono N, Hashimoto T. Molecular analysis of peroxisomal $\beta$-oxidation enzymes in infants with Zellweger syndrome and Zellweger-like syndrome: further heterogeneity of the peroxisomal disorder. Clin Chim Acta $1988 ; 172: 65-76$.

17. Poulos A, van Crugten C, Sharp P, et al. Prenatal diagnosis of Zellweger syndrome and related disorders: impaired degradation of phytanic acid. Eur J Pediatr 1986; 145 : 507-10.

18. Schutgens RBH, Wanders RJA, Heymans HSA, et al. Zellweger syndrome : biochemical procedures in diagnosis, prevention and treatment. J Inher Metab Dis 1987 ; 10 (suppl. 1) : 33-5.

19. Heymans HSA, Oorthuys JWE, Nelck G, Wanders RJA, Schutgens RBH. Rhizomelic chondrodysplasia punctata : another peroxisomal disorder. N Engl J Med 1985 ; 313 : 187 8.

20. Moser HW, Naidu S, Kuman Am, Rosenbaum AR. The adrenoleukodystrophies. CRC Critical Reviews Neurolobiology 1987 ; 3 : 2988.

21. Schaumburg HH, Powers JM, Raine CS, Suzuki K, Richardson EP. Adrenoleukodystrophy. A clinical and pathological study of 17 cases. Arch Neurol 1975 ; 32 : 577-91.

22. Singh I, Moser AE, Goldfischer S, Moser $\mathrm{HW}$. Lignoceric acid is oxidized in the peroxisomes: implications for the Zellweger cerebro-hepato-renal syndrome and adrenoleukodystrophy. Proc Natl Acad Sci USA $1984 ; 81$ : 4203-7.

23. Migeon BR, Moser HW, Moser AB, Axelman J, Sillence D, Norum RA. Adrenoleukodystrophy : evidence for X linkage, inactivation and selection favorizing the mutant allele in heterozygous cells. Proc Natl Acad Sci USA 1981 ; 78: 5066-70.

24. Aubourg PR, Sack GH, Meyers DA, Lease JJ, Moser HW. Linkage of adrenoleukodystrophy to a polymorphic DNA probe. Ann Neurol 1987 ; 21 : 349-52.

25. Moser AB, Borel J, Odone A, et al. A new dietary therapy for adrenoleukodystrophy: biochemical and preliminary clinical results in 36 patients. Ann Neurol 1987 ; 21 : 240-9.

26. Poll-The BT, Roels F, Ogier H, et al. A new peroxisomal disorder with enlarged peroxisomes and a specific deficiency of acylCoA oxidase (pseudo-neonatal adrenoleukodystrophy). Am J Hum Genet 1988; 42 : 422 34.

27. Goldfischer S, Collins J, Rapin I, et al. Pseudo-Zellweger syndrome: deficiencies in several peroxisomal oxidative activities. J Pediatr 1986; 108: 25-32.

28. Schram AW, Goldfischer S, Van Roer mund CWT, et al. Human peroxisomal 3oxoacyl-coenzyme A thiolase deficiency. Proc Natl Acad Sci USA 1987; 84 : 2494-6.

29. Danpure CJ, Jennings PR. Peroxisomal alanine: glyoxylate aminotransferase deficiency in primary hyperoxaluria type I. FEBS
Les peroxysomes sont morphologiquement absents dans quelques hépatocytes et dans d'autres ils ont un aspect anormal (voir Tableau II). On ignore totalement la nature de la lésion moléculaire causale de cette affection mystérieuse qui appartient à un groupe de complémentation autonome.

Les affections des groupes I et II ne comportent à l'heure actuelle aucune possibilité thérapeutique curative ou préventive.

Groupe III : affections à présentation neurologique prédominante comportant un seul déficit biochimique. - L'adrénoleucodystrophie liée à l'X $(A L D)$. Il s'agit probablement du désordre le plus fréquent du métabolisme peroxysomial. C'est une maladie dégénérative dont l'expression phénotypique est très variable, même au sein de la même famille [20].

La forme la plus fréquente est l'ALD juvénile, qui débute en général entre cinq et neuf ans. Dans $85 \%$ des cas, la maladie est caractérisée par l'apparition de troubles du comportement, une détérioration intellectuelle, une diminution de l'acuité visuelle, une surdité, des signes cérébello-spastiques et plus rarement des convulsions. Des signes d'insuffisance surrénale clinique (mélanodermie, hypoglycémie) ou biologique peuvent précéder, accompagner ou suivre l'altération neurologique. Une fois installée, la symptomatologie neurologique progresse rapidement vers une détérioration aboutissant à un état végétatif en deux ans. Le scanner cérébral montre à un stade évolué une image caractéristique reflétant la démyélinisation, surtout dans les régions occipitales et pariétales postérieures. L'étude anatomopathologique montre une démyélinisation intense, une réponse inflammatoire périvasculaire et des inclusions lipidiques dans la substance blanche cérébrale et le cortex surrénal [21] correspondant à des acides gras à très longue chaîne sous forme libre ou estérifiée au cholestérol.

A côté de cette forme juvénile, il existe d'une part la forme d'adrénomyéloneuropathie (AMN) et d'autre part la forme ne comportant qu'une insuffisance surrénale.

L'AMN se manifeste en général au cours de la troisième décennie par une atteinte médullaire et périphérique progressive et une insuffisance surrénale et gonadotrope. Les fonctions intellectuelles sont le plus souvent préservées ou se détériorent tardivement.

Les femmes hétérozygotes peuvent également avoir des signes cliniques proches de la symptomatologie de l'AMN.

L'anomalie biochimique dans tous les cas concerne une accumulation d'acides gras saturés à très longue chaîne dans le plasma, le cerveau, les surrénales et les fibroblastes, probablement secondaire à un déficit en lignocéroyl-CoA synthétase [22]. En revanche, les taux plasmatiques des acides biliaires précurseurs demeurent normaux.

En microscopie électronique, les peroxysomes hépatiques sont normaux.

Le gène muté, responsable de cette maladie, est situé sur le bras long de la région Xq28 du chromosome $X$ [23]. Les études dans des familles ALD/AMN suggèrent une liaison génétique entre ALD et un trouble de la vision des couleurs [24].

Des études préliminaires indiquent que l'utilisation d'un régime alimentaire pauvre en acides gras à très longue chaîne et riche en acide glycérol trioléate diminue les taux plasmatiques de l'acide lignocéroïque [25]. Une tentative de greffe de moelle effectuée dans une forme neurologiquement avancée n'a pas donné de résultats significatifs.

Le diagnostic anténatal est possible par l'étude des acides gras à très longue chaîne sur prélèvement de trophoblaste ou amniocytes cultivés et peut être également réalisé par l'analyse d'ADN. Le dépistage des hétérozygotes est également possible par ces techniques.

- Le déficit en acyl-CoA oxydase peroxysomiale (la pseudo-adrénoleucodystrophie néonatale). Cliniquement les deux patients connus ont un tableau identique à celui de l'ALDN [26]. Dans cette affection, l'accumulation des acides gras à très longue chaîne est en rapport avec un déficit isolé en acyl-CoA oxydase. Les acides biliaires précurseurs ne s'accumulent pas et la biosynthèse des plasmalogènes est normale. Comme dans le pseudo-Zellweger, les peroxysomes hépatiques sont présents et même de 
taille augmentée.

- Le déficit en 3-cétothiolase peroxysomiale (le pseudo-syndrome de Zellweger). Ce désordre a été jusqu'à présent observé chez un seul malade. Celui-ci, une fille, présentait un phénotype clinique remarquablement similaire au syndrome de Zellweger [27]. Cependant, les peroxysomes hépatiques étaient augmentés en nombre et en taille et la biosynthèse des plasmalogènes était normale. Dans cette maladie, il existe une accumulation des acides gras à très longue chaîne due à un déficit spécifique de la 3-cétothiolase peroxysomiale [28]. Cette déficience enzymatique affecte secondairement d'autres étapes de la $\beta$-oxydation peroxysomiale (acides di- et tri-hydroxycoprostanoïques).

Dans cette affection, comme dans la précédente, le diagnostic anténatal est possible par la mise en évidence de l'accumulation d'AGTLC. Il n'y a, à l'heure actuelle, aucune thérapeutique.

- A côté de ces trois désordres bien définis du groupe III, il existe d'autres conditions dont l'identité exacte ou même la nature peroxysomiale demeurent sujettes à discussion.

C'est le cas de l'acidémie pipécolique isolée dont on ne connaît pas le déficit enzymatique. C'est également le cas de la maladie de Refsum classique parfaitement définie sur le plan clinique, biochimique (accumulation d'acide phytanique) et enzymologique (déficit en phytanate $\alpha$-hydroxylase), mais pour laquelle on discute encore de la localisation intracellulaire exacte de la voie catabolique de l'acide phytanique.

Groupe IV : affections non neurologiques à déficit biochimique unique. Deux affections très différentes composent ce groupe.

- L'hyperoxalurie primaire de type I (acidurie glycolique) liée à un déficit en alanine: glyoxylate aminotransférase peroxysomiale hépatique [29] est caractérisée par des dépôts d'oxalate de calcium responsables notamment d'une insuffisance rénale progressivement fatale. Il existe une variété sensible à la pyridoxine, cofacteur de l'enzyme déficitaire. Dans les formes résistantes à la vitamine $\mathrm{B}_{6}$, une possibilité thérapeutique consiste en $\mathrm{m} / \mathrm{s} n^{\circ} 9$ vol. 4 , novembre 88 une double greffe de foie et de rein.

- L'acatalasémie est un défaut génétique rare caractérisé par une déficience de la catalase circulante. A l'exception d'une susceptibilité aux infections orales (maladie de Takahara) cette maladie a peu de manifestations cliniques. Il faut noter que la catalase hépatique a une activité normale.

\section{Les désordres peroxysomiaux secondaires}

A côté des anomalies génétiques cidessus décrites, des anomalies peroxysomiales accompagnent souvent une pathologie mitochondriale héréditaire ou acquise. Il peut s'agir de prolifération de la population peroxysomiale, d'une autophagie des peroxysomes ou d'un catabolisme réduit des acides gras à très longue chaîne. Quand la nature primaire d'un désordre peroxysomial est douteuse, il est important d'étudier les acides organiques urinaires pour éventuellement détecter une maladie mitochondriale sous-jacente.

\section{Conclusion}

Les anomalies héréditaires des peroxysomes constituent un nouveau groupe d'affections qui soulèvent, à l'heure actuelle, de nombreuses questions non résolues. Parmi celles-ci, le rôle physiologique du métabolisme peroxysomial des acides gras, et en particulier des AGTLC et des acides dicarboxyliques, ses relations avec la $\beta$-oxydation mitochondriale et plus généralement les interrelations métaboliques de l'économie énergétique intracellulaire sont parmi les plus intrigants. Ces affections soulèvent aussi la question du rôle des peroxysomes dans la synthèse des molécules complexes telles que les plasmalogènes et de leur utilisation dans les structures cellulaires spécialisées telles que les membranes par exemple. Enfin sur le plan génétique, ces curieux organites qui ne comportent pas d'ADN constituent un remarquable modèle d'études sur les mécanismes d'intégration et la régulation de protéines enzymatiques à l'intérieur d'une structure intracellulaire différenciée

\section{Summary}

Peroxisomes, which are widely distributed in mammalian tissues, play an important role in cellular metabolism. New peroxisomes arise from division of preexisting peroxisomes after the posttranslational import of newly synthesized proteins. Most peroxisomal proteins are synthesized at their final sizes, without cleavable targeting peptides. Peroxisomes contain a fatty acid $\beta$-oxidation system with significant differences from mitochondrial $\beta$-oxidation and is particularly important in the oxidation of very long chain fatty acids and the side chain of cholesterol. Peroxisomes catalyze the initial reactions in the biosynthesis of plasmalogens, which are important phospholipid constituents of cell membranes. Peroxisomal respiration consists of the formation of $\mathrm{H}_{2} \mathrm{O}_{2}$ by a variety of oxidases and the decomposition of the $\mathrm{H}_{2} \mathrm{O}_{2}$ by peroxisomal catalase. A dysfunction of peroxisomes can have serious clinical consequences. An increasing number of genetic diseases with an inborn error of peroxisomal metabolism has recently been recognized, allowing genetic counseling and prenatal diagnosis. Recent data concerning the primary defect in peroxisomal disorders lacking morphologically detectable peroxisomes suggest that peroxisomal membranes may be assembled in these diseases, but that there is a defect in the mechanism for importing proteins into these "ghosts». Precise clinical, biochemical and morphological investigations of peroxisomal disorders have contributed and still contribute to understand the physiological role of peroxisomes.

\section{TIRÉS A PART}

B.T. Poll-The. 